\title{
Playing games with EPR-type experiments
}

\author{
Azhar Iqbal \\ Department of Mathematics, University of Hull, Hull, HU6 7RX, UK
}

\begin{abstract}
An approach towards quantum games is proposed that uses the unusual probabilities involved in EPR-type experiments directly in two-player games.
\end{abstract}

Key words: Quantum games; Local hidden variable; Bell-CHSH inequality; negative probability measure.

\section{Introduction}

The emerging field of quantum games [1, 2, 3, 4, 5, 6, 7, 8, 9, 10, 11, 12, 13, 14, 15, 16, 17 [18, 19, 20, 21, 22, 23, 24 has attracted increasing attention during recent years. Some authors 6] 8. 23. 17. have pointed out that, in certain cases, it is also possible to construct classical description of quantum games, resulting in the presently continuing debate [25. 23, 17] about their true quantum content and character.

Game theory is based on, and extensively uses, the theory of probability [26]. The peculiar probabilities arising in the EPR-type experiments [27, 28, 29, 30] are considered the basis of arguments for quantum non-locality [31. Sometimes, these probabilities are also presented as the most convincing demonstration of how quantum and classical mechanics differ from each other. The motivation behind EPR-type experiments is the EPR paradox 27. In an attempt to resolve the EPR paradox Mückenheim 32 in 1982 made use of negative probability functions. Moreover, recent years have witnessed explicit proofs $33,34,35$ showing how certain probability measures involved in some local hidden variable (LHV) models of the EPR paradox attain negative values. Quoting Mückenheim [36] "Kolmogorov's axiom may hold or not; the probability for the existence of negative probabilities is not negative." Also, Feynman [37, 38, once cleverly anticipated 34, 35. that "The only difference between the classical and quantum cases is that in the former we assume that the probabilities are positive-definite." The physical meaning of the negative probability measures is indeed far from obvious and this particular attempt to resolve the EPR paradox is taken [39] "as unattractive as all others."

In present paper we adapt a positive attitude towards negative probabilities by observing that, in spite of their unattractiveness, they seem to have undisputed value in terms of providing an indication to what can be taken as the true quantum character in certain quantum mechanical experiments. Secondly, the negative probabilities, though labelled as unattractive, have a potential to provide an alternative, and perhaps much shorter, route to construction of simple examples of quantum games. These constructions designed for the physical implementation, and realization, of quantum games will, of course, have the EPR-type experiments as their underlying physical structure.

The approach towards quantum games, developed in this paper, can be divided into two steps. In the first step, elementary probability theory is used to analyze a hypothetical physical implementation of a bi-matrix game, that is motivated by, and has close similarities with, the experimental set-up of the EPR-type experiments. Looking from a distance, this analysis can also be taken as a procedure that re-defines the classical game in a way that justifies and opens the way towards the next step in the present approach. In the second step the peculiar probabilities, coming out of the EPR-type experiments are introduced to see their resulting effects on players' payoff functions and solutions of the game. 
Apart from a being a short-cut towards demonstration and construction of simple quantum games, to us this approach seems to be in demand presently both in game theory and economics [14. Recent years have witnessed serious efforts to entertain the methods and notation of quantum mechanics in these domains. In our view, in spite of these developments, it remains a fact that in these domains the concepts of wavefunction, ket bra notation, and Hermitian operators are still associated with an alien identity; with quantum mechanics believed to be their only right place. Present paper tries to fill in this gap by looking at how quantum games can also be understood by using the peculiar probabilities that appear in certain well-known quantum mechanical experiments, without recourse to the mathematical tools of quantum mechanics. In other words, we try to show how the unusual probabilities in EPR paradox have a potential to leave their mark on game theory.

The rest of this paper is organized as follow. Section (2) compares playing bi-matrix game with the setting of EPR-type experiments to motivate a four-coin physical implementation of a bi-matrix game. Section (3) develops such a hypothetical physical implementation. Section (4) builds up on the construction of the previous section to look at how the game is affected when, instead of four coins, two correlated particles are used to play the game.

\section{Physical implementation of a bi-matrix game}

We consider a two-player two-strategy non-cooperative game that is given as a bi-matrix. Two players Alice and Bob are not allowed to communicate and each player has to go for one of the two available strategies. A usual physical implementation of this game consists of giving two coins to Alice and Bob, each receiving one. Both receive the coin in head state. Each player plays his/her strategy that consists of flipping/not flopping the coin. Afterwards, the players return their coins to a referee. The referee observes the coins and rewards the players according to the strategies they have played and the game under consideration.

Consider now the well-known setting of EPR-type experiments. Once again, Alice and Bob are spatially separated and are unable to communicate with each other. Both receive one half of a pair of particles that originate from a common source. In an individual run both choose one from the two options (strategies) available to him/her. The strategies are usually two directions in space along which measurements can be made. Each measurement generates +1 or -1 as the outcome, which can be associated to head and tail states of a coin, respectively. Experimental results are recorded for a large number of individual runs of the experiment.

Apparent similarities between the two-coin physical implementation of a bi-matrix game and EPR-type experiments can immediately be noticed. The similarities hint to use EPR-type experiments to play bi-matrix games. However, before moving further along that direction, following observations are made:

1. In two-coin implementation of a bi-matrix game, a player knows the head or tail state of his/her coin after he/she has played his/her strategy.

2. In EPR-type experiment when a player decides his/her strategy, as one of two available directions along which a measurement is to be made, he/she does not know whether the measurement is going to result in +1 or -1 , until the measurement has actually been made.

It shows that a two-coin physical implementation of a bi-matrix game is not a right analogy to EPR-type experiments. In an individual run of the EPR-type experiment each player has to chose from one of two available directions. After the player makes a choice between the two directions, the measurement generates +1 or -1 as outcome. It motivates a four-coin implementation of the game. 


\section{Bi-matrix games with four coins}

A bi-matrix game can also be played using four coins instead of the two described above. A procedure that physically implements the game with four coins is as follows. Its motivation comes from the EPR-type experiments and it serves to make possible, in the next step, a smoother transition towards a situation when the same game is played using those experiments.

The players Alice and Bob are given two coins each. It is not known, and it does not matter, whether the given coins are in head or tail states. Before the game starts the referee announces that a player's strategy is to choose one out of the two coins in his/her possession. After playing their strategies, the players give the two chosen coins, representing their strategies, to the referee. The referee tosses both the coins together and records the outcomes. The tossing of the coins is repeated a large number of times, while each player plays his/her strategy each time he/she is asked to choose one of the two coins in his/her possession. After a large number of individual runs the referee rewards the players according to the strategies they have played and the probability distributions followed by the four coins during their tossing.

After stating the general idea, we consider in the following an example of a symmetric bi-matrix game.

$$
\begin{aligned}
& \text { Bob } \\
& \dot{X}_{1} \quad \dot{X}_{2} \\
& \text { Alice } \begin{array}{ccc}
X_{1} & (K, K) & (L, M) \\
& X_{2}
\end{array}\left(\begin{array}{ll}
(M, L) & (N, N)
\end{array}\right)
\end{aligned}
$$

where $X_{1}, X_{2}, \dot{X}_{1}$ and $\dot{X}_{2}$ are the players' strategies. Entries in braces are Alice's and Bob's payoffs, respectively.

We want to physically implement the game (1) using repeated tossing of four coins which follows the following probability distribution

$$
\begin{aligned}
& \text { Bob } \\
& \dot{S}_{1} \quad \dot{S}_{2} \\
& \begin{array}{llll}
H & T & H & T
\end{array} \\
& \begin{array}{lll}
\text { Alice } & S_{1} & H \\
& & T
\end{array}\left(\begin{array}{ll}
p_{1} & p_{2} \\
p_{3} & p_{3}
\end{array}\right) \quad\left(\begin{array}{ll}
p_{5} & p_{6} \\
p_{7} & p_{8}
\end{array}\right) \\
& S_{2} \quad \begin{array}{c}
H \\
T
\end{array}\left(\begin{array}{cc}
p_{9} & p_{10} \\
p_{11} & p_{12}
\end{array}\right) \quad\left(\begin{array}{ll}
p_{13} & p_{14} \\
p_{15} & p_{16}
\end{array}\right)
\end{aligned}
$$

where, for example, $S_{1}$ is Alice's pure strategy to "always select the coin 1" etc. The pure strategies $S_{2}, S_{1}$ and $S_{2}$ can similarly be interpreted. Also, $H \sim$ Head and $T \sim$ Tail and, for obvious reasons, we have

$$
\sum_{1}^{4} p_{i}=\sum_{5}^{8} p_{i}=\sum_{9}^{12} p_{i}=\sum_{13}^{16} p_{i}=1
$$

In construction of the four-coin statistics (2) following points should be taken into consideration.

1. The statistics (2) may convey the impression that in an individual run both players forward both of their coins to the referee who tosses the four coins together. In fact, in an individual run the referee tosses only two coins. The statistics (2) are generated under the assumption that there is randomness involved in players' strategies to go for one or the other coin.

2. Associated with the above impression is the fact that, in every individual run, the statistics (2) assign head or tail states to the two coins that have not been tossed. So that, in each individual run two tosses are counterfactual. 
Because counterfactual reasoning is involved in the derivation of Bell-CHSH inequality, some authors [40, 41, 42] have argued that quantum non-locality 31] (or locality) does not follow from the violation of the Bell-CHSH inequality in EPR-type experiments. With reference to our coin game counterfactual reasoning means that two coins, out of four, are not tossed in each individual turn, but still these untossed coins are assigned head or tail states in the mathematical steps used in the derivation of Bell-CHSH inequality. This assignment is often justified under the label of realism. In EPR-type experiments the measurements along two directions, for each player, do not commute with each other i.e. joint measurements can not be made. Based on this fact some arguments [43, 44, 45] say that the reasons for the violation of Bell-CHSH inequality in EPR-type experiments reside only in non-commutative nature of the quantum measurements involved.

To play the game (11) with four-coin statistics (2), we assume the referee has the following recipe $^{1}$ to reward the players.

$$
\left.\begin{array}{c}
P_{A}\left(S_{1}, \dot{S}_{1}\right)=K p_{1}+L p_{2}+M p_{3}+N p_{4} \\
P_{A}\left(S_{1}, \dot{S}_{2}\right)=K p_{5}+L p_{6}+M p_{7}+N p_{8} \\
P_{A}\left(S_{2}, \dot{S}_{1}\right)=K p_{9}+L p_{10}+M p_{11}+N p_{12} \\
P_{A}\left(S_{2}, \dot{S}_{2}\right)=K p_{13}+L p_{14}+M p_{15}+N p_{16}
\end{array}\right\}
$$

Where $P_{A}\left(S_{1}, \dot{S}_{2}\right)$, for example, is Alice's payoff when she plays $S_{1}$ and Bob plays $\dot{S}_{2}$. The corresponding payoff expressions for Bob can be found by the transformation $L \leftrightarrows M$ in Eqs. (4). The recipe, of course, makes sense if repeated tosses are made with four coins. Because $S_{1}, S_{2}, S_{1}$ and $S_{2}$ are taken as players' pure strategies; a mixed strategy for Alice, for example, is convex linear combination of $S_{1}$ and $S_{2}$.

We now find constraints on four-coin statistics (2) such that each equation in (4) represents mixed strategy payoff for the bi-matrix game (1), that can be written in bi-linear form. To allow this interpretation for the payoffs (44) four probabilities $r, s, \dot{r}$ and $s$ are required that can give a bi-linear representation to the payoffs (4) i.e.

$$
\left.\begin{array}{l}
P_{A}\left(S_{1}, \dot{S}_{1}\right)=K r \dot{r}+L r(1-\dot{r})+M \dot{r}(1-r)+N(1-r)(1-\dot{r}) \\
P_{A}\left(S_{1}, S_{2}\right)=K r \dot{s}+L r(1-\dot{s})+M \dot{s}(1-r)+N(1-r)(1-\dot{s}) \\
P_{A}\left(S_{2}, \dot{S}_{1}\right)=K s \dot{r}+L s(1-\dot{r})+M \dot{r}(1-s)+N(1-s)(1-\dot{r}) \\
P_{A}\left(S_{2}, \dot{S}_{2}\right)=K s \dot{s}+L s(1-\dot{s})+M \dot{s}(1-s)+N(1-s)(1-\dot{s})
\end{array}\right\}
$$

It then allows to make the association

$$
S_{1} \sim r, \quad S_{2} \sim s, \quad \dot{S}_{1} \sim \dot{r}, \quad \dot{S}_{2} \sim \dot{s}
$$

where $r, s, \dot{r}$ and $\dot{s}$ are the probabilities of heads for coins $S_{1}, S_{2}, \dot{S}_{1}$ and $\dot{S}_{2}$, respectively. In case a consistent set of these four probabilities is found, each equation in (4) can be interpreted in terms of a mixed strategy game between the two players. So that, the four pairs

$$
\left(r, r^{\prime}\right), \quad(r, \dot{s}), \quad(s, \dot{r}), \quad\left(s, s^{\prime}\right)
$$

represent the four possible situations that may result when each player has got two strategies to choose from. For example, the strategy pair $\left(S_{1}, S_{2}\right)$ is associated with the pair $(r, s)$ and it corresponds to the mixed strategy game given as

$$
\begin{aligned}
& \text { Bob } \\
& \text { Alice } \begin{array}{c}
r \\
(1-r)
\end{array}\left(\begin{array}{cc}
\dot{s} & (1-\dot{s}) \\
(K, K) & (L, M) \\
(M, L) & (N, N)
\end{array}\right)
\end{aligned}
$$

In this game Alice plays $S_{1}$ with probability of heads $r$, and Bob plays $S_{2}$ with probability of heads $s$. The other equations in (5) can be given similar interpretation.

\footnotetext{
${ }^{1}$ The recipe [4 is not unique and others may be suggested. Any recipe is justified if it is able to reproduce the game under consideration within the description of the statistical experiment involved.
} 
We now find constraints on four-coin statistics (2) that make the payoffs of Eq. (4) identical to the bi-linear payoffs of Eq. (5) for any real numbers $K, L, M$ and $N$. A comparison of these equations shows that it happens when $r, s, \dot{r}$ and $\dot{s}$ depend on $p_{i}$, for $16 \geqslant i \geqslant 0$, as follows

$$
\left.\begin{array}{cccc}
r \dot{r}=p_{1}, & r(1-\dot{r})=p_{2}, & \dot{r}(1-r)=p_{3}, & (1-r)(1-\dot{r})=p_{4} \\
r \dot{s}=p_{5}, & r(1-\dot{s})=p_{6}, & \dot{s}(1-r)=p_{7}, & (1-r)(1-\dot{s})=p_{8} \\
s \dot{r}=p_{9}, & s(1-\dot{r})=p_{10}, & \dot{r}(1-s)=p_{11}, & (1-s)(1-\dot{r})=p_{12} \\
s \dot{s}=p_{13}, & s(1-\dot{s})=p_{14}, & \dot{s}(1-s)=p_{15}, & (1-s)(1-\dot{s})=p_{16}
\end{array}\right\}
$$

The probabilities $r, s, \dot{r}$ and $s$ can be read from (9) as

$$
r=p_{1}+p_{2}, \quad s=p_{9}+p_{10}, \quad \dot{r}=p_{1}+p_{3}, \quad \dot{s}=p_{5}+p_{7}
$$

provided that $p_{i}$ satisfy

$$
\left.\begin{array}{c}
p_{1}+p_{2}=p_{5}+p_{6}, \quad p_{1}+p_{3}=p_{9}+p_{11} \\
p_{9}+p_{10}=p_{13}+p_{14}, \quad p_{5}+p_{7}=p_{13}+p_{15}
\end{array}\right\}
$$

With the defining relations (10), and the constraints (11) on the four-coin statistics, each pair in $\left(S_{1}, S_{1}\right),\left(S_{1}, S_{2}\right),\left(S_{2}, S_{1}\right)$ and $\left(S_{2}, S_{2}\right)$ gains interpretation of a mixed strategy game. The correspondence (6) means that, for example, Alice's payoffs read as

$$
\left.\begin{array}{ll}
P_{A}\left(S_{1}, \dot{S}_{1}\right)=P_{A}(r, \dot{r}), & P_{A}\left(S_{1}, \dot{S}_{2}\right)=P_{A}\left(r, s^{\prime}\right) \\
P_{A}\left(S_{2}, \dot{S}_{1}\right)=P_{A}(s, \dot{r}), & P_{A}\left(S_{2}, \dot{S}_{2}\right)=P_{A}\left(s, s^{\prime}\right)
\end{array}\right\}
$$

Now, suppose $(s, s)$ is a Nash equilibrium (NE) i.e.

$$
\left\{P_{A}\left(s, s^{\prime}\right)-P_{A}(r, s)\right\} \geqslant 0, \quad\left\{P_{B}\left(s, s^{\prime}\right)-P_{B}\left(s, \dot{r}^{\prime}\right)\right\} \geqslant 0
$$

Using Eqs. (5) one gets

$$
\left.\begin{array}{l}
P_{A}(s, \dot{s})-P_{A}(r, \dot{s})=(s-r)\{(K-M-L+N) \dot{s}+(L-N)\} \geqslant 0 \\
P_{B}(s, \dot{s})-P_{B}(s, \dot{r})=(\dot{s}-\dot{r})\{(K-M-L+N) s+(L-N)\} \geqslant 0
\end{array}\right\}
$$

Consider the game of prisoners' dilemma (PD) which is produced when $M>K>N>L$ in the matrix (11). We select our first representation of PD by taking

$$
K=3, L=0, M=5 \text { and } N=1
$$

and the inequalities (14) are reduced to

$$
0 \geqslant(s-r)(1+\dot{s}), \quad 0 \geqslant(\dot{s}-\dot{r})(1+s)
$$

Now from (14) the pair $(s, s)$ is a NE when both inequalities in (16) are true for all $r, \dot{r} \in[0,1]$. Because $(1+s) \geqslant 1$ and $(1+s) \geqslant 1$, it produces $s=s=0$ as the equilibrium. In present set-up to play PD this equilibrium appears if, apart from the constraints of Eqs. (10 11), we also have

$$
s=p_{9}+p_{10}=0, \quad s=p_{5}+p_{7}=0
$$

which are other constraints on the four-coins statistics (2) to hold true if the PD produces the NE $(s, s)=(0,0)$.

The above analysis can be reproduced for other probability pairs in (7). For example, when $(r, s)$ is NE i.e.

$$
\left\{P_{A}\left(r, s^{\prime}\right)-P_{A}\left(s, s^{\prime}\right)\right\} \geqslant 0, \quad\left\{P_{B}\left(r, s^{\prime}\right)-P_{B}\left(r, r^{\prime}\right)\right\} \geqslant 0
$$

and we again get $\left(r, s^{\prime}\right)=(0,0)$. The relations (10) though now say that it will exist as a NE when

$$
p_{1}+p_{2}=0, \quad p_{5}+p_{7}=0
$$


which should, of course, be true along with the relations (11). That is, in order to reproduce a particular NE in the bi-matrix game the probabilities of heads of the four coins representing the game need to be fixed. Also, from the bi-linear payoffs (5) it is clear that at the equilibria $\left(s, s^{\prime}\right)=(0,0)$ and $\left(r, s^{\prime}\right)=(0,0)$ the reward for both the players is $N$.

Summarizing, we have shown that when the four-coin statistics (2) satisfy the constraints of Eqs. (3 11), the payoffs (4) can be interpreted in terms of a mixed strategy version of a bi-matrix game. In this setting four strategies $S_{1}, S_{2}, S_{1}$ and $S_{2}$ available to the players are associated with the probabilities $r, s, \dot{r}$ and $\dot{s}$, respectively. This association allows to interpret the payoff recipe of Eq. (4) in terms of a mixed strategy game. We showed that when $r, s, \dot{r}$ and $\dot{s}$ are expressed in terms of the probabilities $p_{i}$ for $16 \geqslant i \geqslant 1$ (as it is the case in the Eqs. (10)) the bi-linear payoffs (5) become identical to the payoffs of the Eq. (4). This procedure is designed to re-express playing a bi-matrix game with four coins in a way that choosing which coin to toss is a player's strategy.

\section{Games with perfectly correlated particles}

The re-expression of playing a bi-matrix game in terms of a four-coin tossing experiment, performed between two players, opens the way to see what happens when the four-coin statistics become correlated. Especially, what if the correlations go beyond what is achievable with the so-called classical 'coins'.

Presently, there appears general agreement in quantum physics community that the EPR-type experiments, performed on correlated pairs of particles, violate the predictions of LHV models. Negative probabilities are found to emerge when certain LHV models are forced to predict the experimental outcomes of EPR-type experiments. For example, Han, Hwang and Koh [33] showed the need for negative probabilities when explicit solutions can reproduce quantum mechanical predictions for some spin-measurement directions for all entangled states. In Han et al.'s analysis a special basis is used to show the appearance of negative probabilities for a class of LHV models.

Rothman and Sudarshan [35] demonstrated that quantum mechanics does predict a set of probabilities that violate the CHSH inequality; however these probabilities are not positive-definite. Nevertheless, they are physically meaningful in that they give the usual quantum-mechanical predictions in physical situations. Rothman and Sudarshan observed that all derivations of Bell's inequalities assume that LHV theories produce a set of positive-definite probabilities for detecting a particle with a given spin orientation.

Using a similar approach, Cereceda [34] proved independently the necessity of negative probabilities in all instances where the predictions of the LHV model are made to violate the Bell-CHSH inequality. Interestingly, Cereceda's proof does not rely on any particular basis states or measurement directions. In the concluding section of his paper Cereceda analyzes the case of pairs of particles that have perfect correlation between them. He then proceeds to show the necessity of negative probabilities for those pairs.

The necessity of negative probability measures, to explain the experimental outcomes in EPRtype experiments, motivates questions about the effects and consequences these may have on solution of a game which is physically implemented using such experiments. This question can be expressed as follows. What happens to the players' payoffs and solutions of a game that is physically implemented using pairs of perfectly correlated particles? It seems quite reasonable to demand that, when the predictions of LHV model agree with the Bell-CHSH inequality, the game attains classical interpretation.

One clear advantage of the above approach towards quantum games appears to be that it is possible to see, without using the machinery of quantum mechanics, how game-theoretical solutions are affected when a game is physically implemented using quantum mechanically correlated pairs of particles.

We follow Cereceda's notation [34] for probabilities in EPR-type experiments designed to test Bell-CHSH inequality. Two correlated particles 1 and 2 are emitted in opposite directions from a common source. Afterwards, each of the particles enters its own measuring apparatus which can 
measure either one of two physical variables at a time. We denote these variables $S_{1}$ or $S_{2}$ for particle 1 and $S_{1}$ or $S_{2}$ for particle 2 . These variables can take possible values of +1 and -1 . The source emits very large number of particle pairs.

To describe this experiment Cereceda 34 considers a deterministic hidden variable model as follows. The model assumes that there exists a hidden variable $\lambda$ for every pair of particles emitted by the source. $\lambda$ has a domain of variation $\Lambda$ and it determines locally (for example, at the common source) the response of the particle to each of the measurements they can be subjected to. It is possible to partition the set of all $\lambda$ into 16 disjoint subsets $\Lambda_{i}$ (with respect to probability measure $m_{i}$ ) according to the outcomes of the four possible measurements, $S_{1}$ or $S_{2}$ for particle 1 and $S_{1}$ or $S_{2}$ for particle 2 . Table 1 is reproduced from Cereceda's paper. It shows the 16 rows characterizing the subsets $\Lambda_{i}$. The $i$ th row gives the outcome of different measurements when the particle pair is described by a hidden variable pertaining to the subset $\Lambda_{i}$.

Table 1: The set $\Lambda$ is partitioned into 16 possible subsets. The hidden variables in each subset $\Lambda_{i}$ uniquely determine the outcomes for each of the four possible single measurements $S_{1}, S_{1}, S_{2}$, and $\dot{S}_{2}$. The table is reproduced from the Ref. [34].

$\begin{array}{cccccc}\text { Subset of } \Lambda & S_{1} & \dot{S}_{1} & S_{2} & \dot{S}_{2} & \text { Probability measure } \\ \Lambda_{1} & + & + & + & + & m_{1} \\ \Lambda_{2} & + & + & + & - & m_{2} \\ \Lambda_{3} & + & + & - & + & m_{3} \\ \Lambda_{4} & + & + & - & - & m_{4} \\ \Lambda_{5} & + & - & + & + & m_{5} \\ \Lambda_{6} & + & - & + & - & m_{6} \\ \Lambda_{7} & + & - & - & + & m_{7} \\ \Lambda_{8} & + & - & - & - & m_{8} \\ \Lambda_{9} & - & + & + & + & m_{9} \\ \Lambda_{10} & - & + & + & - & m_{10} \\ \Lambda_{11} & - & + & - & + & m_{11} \\ \Lambda_{12} & - & + & - & - & m_{12} \\ \Lambda_{13} & - & - & + & + & m_{13} \\ \Lambda_{14} & - & - & + & - & m_{14} \\ \Lambda_{15} & - & - & - & + & m_{15} \\ \Lambda_{16} & - & - & - & - & m_{16}\end{array}$

The probabilities $p_{i}$ are given below in obvious notation 34 .

$$
\begin{aligned}
p_{1} & \equiv p\left(S_{1}+; \dot{S}_{1}+\right)=m_{1}+m_{2}+m_{3}+m_{4}, \\
p_{2} & \equiv p\left(S_{1}+; \dot{S}_{1}-\right)=m_{5}+m_{6}+m_{7}+m_{8}, \\
p_{3} & \equiv p\left(S_{1}-; \dot{S}_{1}+\right)=m_{9}+m_{10}+m_{11}+m_{12}, \\
p_{4} & \equiv p\left(S_{1}-; \dot{S}_{1}-\right)=m_{13}+m_{14}+m_{15}+m_{16}, \\
p_{5} & \equiv p\left(S_{1}+; \dot{S}_{2}+\right)=m_{1}+m_{3}+m_{5}+m_{7}, \\
p_{6} & \equiv p\left(S_{1}+; \dot{S}_{2}-\right)=m_{2}+m_{4}+m_{6}+m_{8}, \\
p_{7} & \equiv p\left(S_{1}-; \dot{S}_{2}+\right)=m_{9}+m_{11}+m_{13}+m_{15}, \\
p_{8} & \equiv p\left(S_{1}-; \dot{S}_{2}-\right)=m_{10}+m_{12}+m_{14}+m_{16}, \\
p_{9} & \equiv p\left(S_{2}+; \dot{S}_{1}+\right)=m_{1}+m_{2}+m_{9}+m_{10}, \\
p_{10} & \equiv p\left(S_{2}+; \dot{S}_{1}-\right)=m_{5}+m_{6}+m_{13}+m_{14}, \\
p_{11} & \equiv p\left(S_{2}-; \dot{S}_{1}+\right)=m_{3}+m_{4}+m_{11}+m_{12}, \\
p_{12} & \equiv p\left(S_{2}-; \dot{S}_{1}-\right)=m_{7}+m_{8}+m_{15}+m_{16}, \\
p_{13} & \equiv p\left(S_{2}+; \dot{S}_{2}+\right)=m_{1}+m_{5}+m_{9}+m_{13}, \\
p_{14} & \equiv p\left(S_{2}+; \dot{S}_{2}-\right)=m_{2}+m_{6}+m_{10}+m_{14},
\end{aligned}
$$




$$
\begin{aligned}
& p_{15} \equiv p\left(S_{2}-; \dot{S}_{2}+\right)=m_{3}+m_{7}+m_{11}+m_{15} \\
& p_{16} \equiv p\left(S_{2}-; \dot{S}_{2}-\right)=m_{4}+m_{8}+m_{12}+m_{16}
\end{aligned}
$$

Combining Eqs. (3) with Table 1 gives

$$
\sum_{i=1}^{16} m_{i}=1
$$

Continuing with Cereceda's description 34 an example is now considered. Suppose the particle pair is described by a given $\lambda \in \Lambda_{2}$, then the particles must behave as follows. If $S_{1}$ is measured on particle 1 the result will be +1 , if $S_{2}$ is measured on particle 1 the result will be +1 , if $S_{1}$ is measured on particle 2 the result will be +1 , if $S_{2}$ is measured on particle 2 the result will be -1 . Also for each of the plans the results of measurements made on particle 1 are independent of the results of measurements made on particle 2 .

For perfectly correlated particles two of the probabilities $p_{2}$ and $p_{3}$ can be set equal to zero. Physically it means that the results for the joint measurement of two observables, one for each particle, must both be either +1 or -1 . From a physical point of view, it is reasonable to suppose that, for the case in which $p_{2}=0$ and $p_{3}=0$, the probability measures $m_{5}, m_{6}, m_{7}, m_{8}, m_{9}, m_{10}, m_{11}$, and $m_{12}$ also vanish. It can be verified form Eqs. (21) 22). If this is not the case then joint detection events will be generated by the LHV model, which do not happen according to the assumptions made. Cereceda showed that, in this case, when the predictions of the LHV model violate the Bell-CHSH inequality, the negativity of either $m_{4}$ or $m_{13}$ can be proved.

We assume now that the probabilities $p_{i}$ appearing in Eqs. (4) correspond to the LHV model of the EPR-type experiments performed to test Bell-CHSH inequality. Like it is the case with the four-coin tossing experiment, the payoffs (4) can be interpreted as bi-linear payoffs. To do this we use the Eqs. (10, 11) to get

$$
r=p_{1}=p_{5}+p_{6}, \quad r=p_{1}=p_{9}+p_{11}, \quad s=p_{9}+p_{10}, \quad s=p_{5}+p_{7}
$$

But $p_{2}=p_{3}=0$, so that from the first Eq. of (9) we have $r(1-r)=\dot{r}(1-r)$ which gives $p_{1}=0$ or 1 because $r=r^{\prime}=p_{1}$ from (37). We select ${ }^{2} p_{1}=1$. Now, from Eqs. (24) 25) we have $p_{5}+p_{6}=\sum_{i=1}^{8} m_{i}$ and because $\sum_{i=1}^{16} m_{i}=1$ from Eq. (36) and $m_{i}=0$ for $12 \geqslant i \geqslant 5$ we get

$$
\left.\begin{array}{c}
r=\dot{r}=1=m_{1}+m_{2}+m_{3}+m_{4} \\
s=m_{1}+m_{2}+m_{13}+m_{14} \\
s=m_{1}+m_{3}+m_{13}+m_{15}
\end{array}\right\}
$$

With these relations the bi-linear payoffs (15) are written as

$$
\begin{aligned}
& P_{A}\left(S_{1}, \dot{S}_{1}\right)=K \\
& P_{A}\left(S_{1}, \dot{S}_{2}\right)=L+(K-L)\left(m_{1}+m_{3}+m_{13}+m_{15}\right) \\
& P_{A}\left(S_{2}, \dot{S}_{1}\right)=M+(K-M)\left(m_{1}+m_{2}+m_{13}+m_{14}\right) \\
& \left.\begin{array}{c}
P_{A}\left(S_{2}, \dot{S}_{2}\right)=(K-L-M+N) \times \\
\left(m_{1}+m_{2}+m_{13}+m_{14}\right)\left(m_{1}+m_{3}+m_{13}+m_{15}\right)+ \\
(L-N)\left(m_{1}+m_{2}+m_{13}+m_{14}\right)+ \\
(M-N)\left(m_{1}+m_{3}+m_{13}+m_{15}\right)+N
\end{array}\right\}
\end{aligned}
$$

Each of the correlated payoffs (40 41, 42) can be split into two parts i.e.

\footnotetext{
${ }^{2}$ Selecting $p_{1}=0$ makes $p_{4}=1$ because $\sum_{i=1}^{4} p_{i}=1$. It will result in different but analogous expressions for $r, \dot{r}, s$ and $s$ given in terms of $m_{i}$ without affecting the present argument.
} 


$$
\left.\begin{array}{l}
P_{A}\left(S_{1}, \dot{S}_{2}\right)=P_{A_{a}}\left(S_{1}, \dot{S}_{2}\right)+P_{A_{b}}\left(S_{1}, \dot{S}_{2}\right) \\
P_{A}\left(S_{2}, \dot{S}_{1}\right)=P_{A_{a}}\left(S_{2}, \dot{S}_{1}\right)+P_{A_{b}}\left(S_{2}, \dot{S}_{1}\right) \\
P_{A}\left(S_{2}, \dot{S}_{2}\right)=P_{A_{a}}\left(S_{2}, \dot{S}_{2}\right)+P_{A_{b}}\left(S_{2}, \dot{S}_{2}\right)
\end{array}\right\}
$$

where

$$
\begin{aligned}
& P_{A_{a}}\left(S_{1}, \dot{S}_{2}\right)=L+(K-L)\left(m_{1}+m_{3}\right) \\
& P_{A_{b}}\left(S_{1}, S_{2}\right)=(K-L)\left(m_{13}+m_{15}\right) \\
& P_{A_{a}}\left(S_{2}, \dot{S}_{1}\right)=M+(K-M)\left(m_{1}+m_{2}\right) \\
& P_{A_{b}}\left(S_{2}, S_{1}\right)=(K-M)\left(m_{13}+m_{14}\right) \\
& \left.\begin{array}{c}
P_{A_{a}}\left(S_{2}, \dot{S}_{2}\right)=(K-L-M+N)\left(m_{1}+m_{2}\right)\left(m_{1}+m_{3}\right)+ \\
(L-N)\left(m_{1}+m_{2}\right)+(M-N)\left(m_{1}+m_{3}\right)+N
\end{array}\right\} \\
& \left.\begin{array}{c}
P_{A_{b}}\left(S_{2}, \dot{S}_{2}\right)=(K-L-M+N)\left\{\left(m_{1}+m_{2}\right)\left(m_{13}+m_{15}\right)+\right. \\
\left.\left(m_{1}+m_{3}\right)\left(m_{13}+m_{14}\right)+\left(m_{13}+m_{14}\right)\left(m_{13}+m_{15}\right)\right\}+ \\
(L-N)\left(m_{13}+m_{14}\right)+(M-N)\left(m_{13}+m_{15}\right)
\end{array}\right\}
\end{aligned}
$$

The significance of this splitting is that components $P_{A_{b}}\left(S_{1}, \dot{S}_{2}\right), P_{A_{b}}\left(S_{2}, \dot{S}_{1}\right)$ and $P_{A_{b}}\left(S_{2}, \dot{S}_{2}\right)$ of the Alice's payoffs in Eqs. (43) become zero when the predictions of LHV model agree with the Bell-CHSH inequality. Cheon and Tsutsui [17] have also shown a similar splitting using correlated payoff operators whose expectation values are the players' payoffs.

Consider again the PD with the selection of the constants given in (15). Let the game be played using correlated particles, for which substitutions can be made from (38) into the Nash equilibrium condition (16). It gives

$$
0 \geqslant(s-1)(1+s), \quad 0 \geqslant(s-1)(1+s)
$$

where

$$
\begin{aligned}
& s=m_{1}+m_{2}+m_{13}+m_{14} \\
& \dot{s}=m_{1}+m_{3}+m_{13}+m_{15}
\end{aligned}
$$

It can be noticed that

- The predictions of the LHV model agree with the Bell-CHSH inequality. It makes $m_{i} \geqslant 0$ for all $16 \geqslant i \geqslant 1$. Combining it with (38), i.e. $\sum_{i=1}^{4} m_{i}=1$, gives

$$
m_{13}=m_{14}=m_{15}=m_{16}=0
$$

So that, it reduces $s$ and $s$ in (51 52) to

$$
s_{1}=m_{1}+m_{2}, \quad \dot{s}_{1}=m_{1}+m_{3}
$$

- The requirement $\left(s_{1}, s_{1}\right)=(0,0)$ says that when the predictions of the LHV model agree with the Bell-CHSH inequality the pair $(0,0)$ is a NE. Eq. (54) gives

$$
m_{1}=m_{2}=m_{3}=0
$$

Before proceeding to raise a question, we make following observations: 
1. Eqs. (20) to (35) give the probabilities $p_{i}$ in terms of $m_{i}$, for $16 \geqslant i \geqslant 1$, corresponding to the EPR-type experiments. These probabilities satisfy the constraints (11) that emerge when Eqs. (4) are interpreted in terms of bi-linear payoffs of Eqs. (5).

2. The expressions (38) for $r, s, r$ and $s$ are obtained from the corresponding expressions for the coin tossing case (10), while taking into consideration the constraints on probabilities for perfectly correlated particles.

3. The Eqs. (53) 55) together make $s=s=0$ in the definitions (51 52). These definitions correspond to the representation (15) of PD, for which constraints (17) should be true in case $s=s=0$ is a NE when the game is played with coins. It is observed that both $\left(p_{9}+p_{10}\right)$ and $\left(p_{5}+p_{7}\right)$ become zero from the definitions of $p_{i}$ in terms of $m_{i}$ given in Table 1 , when Eqs. (53] [55) both are true. It means that when PD gives $s=s=0$ as an equilibrium the constraints on probabilities become identical in the following two cases:

a) The game is played using repeated tosses with four coins.

b) The game is played with perfectly correlated particles such that the predictions of LHV model agree with the Bell-CHSH inequality.

Bi-matrix games other than $\mathrm{PD}$, presumably with different Nash equilibria, would give rise to different but analogous constraints on the probabilities $s, \dot{s}, r$ and $\dot{r}$. In the light of these observations following question arises immediately. What happens to the Nash conditions (50) when the predictions of LHV model disagree with the Bell-CHSH inequality? To answer it consider the Nash conditions (50) with a substitution from (55). These give

$$
\left.\begin{array}{l}
0 \geqslant\left(s_{2}-1\right)\left(1+s_{2}\right) \\
0 \geqslant\left(s_{2}-1\right)\left(1+s_{2}\right)
\end{array}\right\}
$$

where

$$
s_{2}=m_{13}+m_{14}, \quad \dot{s}_{2}=m_{13}+m_{15}
$$

Now we recall that in Cereceda's analysis $m_{13}$ can take negative value when the predictions of LHV model disagree with the Bell-CHSH inequality. Both $\left(s_{2}-1\right)$ and $\left(\dot{s}_{2}-1\right)$ in (56) remain negative whether $m_{13}$ is positive or negative. Similarly, both $\left(1+s_{2}\right)$ and $\left(1+s_{2}\right)$ remain positive whether $m_{13}$ is positive or negative. Therefore, the Nash conditions (56), that corresponds to the representation (15) of $\mathrm{PD}$, are not violated whether the predictions of the LHV model agree or disagree with the Bell-CHSH inequality.

Players' payoffs at the equilibrium $\left(s_{2}, s_{2}\right)$ can be found from Eq. (49) as

$$
\left.\begin{array}{c}
P_{A_{a}}\left(S_{2}, \dot{S}_{2}\right)=P_{B_{a}}\left(S_{2}, \dot{S}_{2}\right)=N \\
P_{A_{b}}\left(S_{2}, \dot{S}_{2}\right)=(K-L-M+N) s_{2} \dot{s}_{2}+(L-N) s_{2}+(M-N) \dot{s}_{2} \\
P_{B_{b}}\left(S_{2}, \dot{S}_{2}\right)=(K-L-M+N) s_{2} \dot{s}_{2}+(M-N) s_{2}+(L-N) \dot{s}_{2}
\end{array}\right\}
$$

where $s_{2}$ and $s_{2}$ are read from (57). For example, with PD's representation (15), the players' payoffs are obtained from Eqs. 4849) as

$$
\left.\begin{array}{l}
P_{A}\left(S_{2}, \dot{S}_{2}\right)=\dot{s}_{2}\left(4-s_{2}\right)-s_{2}+1 \\
P_{B}\left(S_{2}, \dot{S}_{2}\right)=s_{2}\left(4-\dot{s}_{2}\right)-\dot{s}_{2}+1
\end{array}\right\}
$$

The NE $\left(s_{2}, s_{2}\right)$ in Eqs. (57) corresponds when the predictions of LHV model disagree with the Bell-CHSH inequality. Its defining inequalities (56) show that it exists even when either $s_{2}$ or $s_{2}$ take negative values, which can be realized when $m_{13}$ is negative. So that, the NE in PD's representation (15) can be 'displaced' when the predictions of LHV model disagree with the Bell-CHSH inequality. Here displacement means that either $s_{2}$ or $s_{2}$ can take negative values. However, this extra freedom of assuming negative values does not disqualify $\left(s_{2}, s_{2}\right)$ to exist as a 
NE. From Eq. (59) it can be noticed that $P_{A}\left(S_{2}, S_{2}\right)$ and $P_{B}\left(S_{2}, S_{2}\right)$ can not be greater than 1 when both $s_{2}$ and $s_{2}$ take negative values.

We show now that it may not be the case with another representation of PD. That is, the extra freedom for $s_{2}$ and $s_{2}$ to take negative values, granted when the predictions of the LHV model disagree with Bell-CHSH inequality, leads to disqualification of $\left(s_{2}, s_{2}\right)$ to exist as a NE in that representation of $\mathrm{PD}$.

Consider the PD with a slightly different value assigned to the constant $N$ of the game [17]

$$
K=3, \quad L=0, \quad M=5 \text { and } N=0.2
$$

In this representation the inequalities (14) are reduced to

$$
0 \geqslant(s-r)(1.8 \dot{s}+0.2), \quad 0 \geqslant(\dot{s}-\dot{r})(1.8 s+0.2)
$$

A substitution of $r=\dot{r}=1$ from (38) and then addition of both the inequalities gives

$$
\frac{1}{9}\left\{4\left(s+s^{\prime}\right)+1\right\} \geqslant s s^{\prime}
$$

Suppose that the predictions of LHV model disagree the Bell-CHSH inequality i.e. both $s$ and $s$ are to be replaced by $s_{2}$ and $s_{2}$ in (62)

$$
\frac{1}{9}\left\{4\left(s_{2}+\dot{s}_{2}\right)+1\right\} \geqslant s_{2} \dot{s}_{2}
$$

where $s_{2}$ and $s_{2}$ are given by (57). Interestingly, it is observed that the inequality (63) is violated if $-0.25>\left(s_{2}+s_{2}\right)$ and the NE of Eq. (57) ceases to exist. Of course, it applies to the representation of PD given by (60). Players' payoffs are given as

$$
\left.\begin{array}{l}
P_{A}\left(S_{2}, \dot{S}_{2}\right)=\dot{s}_{2}\left(4.8-1.8 s_{2}\right)+0.2\left(1-s_{2}\right) \\
P_{B}\left(S_{2}, \dot{S}_{2}\right)=s_{2}\left(4.8-1.8 \dot{s}_{2}\right)+0.2\left(1-\dot{s}_{2}\right)
\end{array}\right\}
$$

As it is the case with the first representation of PD, the payoffs $P_{A}\left(S_{2}, \dot{S}_{2}\right), P_{B}\left(S_{2}, \dot{S}_{2}\right)$ can not be greater than 0.2 when both $s_{2}$ and $s_{2}$ taken negative values.

It shows that in the physically implementation of $\mathrm{PD}$, using perfectly correlated particles, the two representations (15)60) behave differently from each other. In representation (15) the disagreement of the predictions of LHV model with the Bell-CHSH inequality leads to a displacement of the NE $(s, s)$ such that $s$ and $s$ can assume negative values. Displacement occurs but $(s, s)$ continues to exist as a NE.

On the other hand, in the representation (60) the disagreement of the predictions of LHV model with the Bell-CHSH inequality leads to the disappearance of the NE $(s, s)$ when both $s$ and $s$ assume negative values and their sum becomes less than -0.25 .

Alert reader may come up with a 'minimalist' interpretation of the present approach as follows. Constraints (1117) are required on four-coin statistics (2) to make $(s, s)=(0,0)$ a NE when the $\mathrm{PD}$ is played in representation (15) with repeated tosses of four coins. When the same game is played with pairs of perfectly correlated particles and the predictions of LHV model disagree with the Bell-CHSH inequality, we can have $\left(p_{9}+p_{10}\right)$ or $\left(p_{5}+p_{7}\right)$ becoming negative which denies (17). If it affects the solution of the game then one can say that it is because of the change in the underlying probabilities of our physical system. In our point of view it is not the question of changing the underlying probabilities. On the other hand, it is a procedure that addresses the question asking what is the true quantum content of quantum game in the following two steps:

1. For perfectly correlated particles, developing an association that guarantees a classical game results when the predictions of LHV model do not violate the Bell-CHSH inequality.

2. With above association retained, how solutions of a game are affected when the LHV model violates the Bell-CHSH inequality. 
When these steps taken into consideration the possibility of construction of a classical game, that reproduces a quantum game, can not be taken as a blow to the subject of quantum games [8]. In our opinion the question quantum game theory asks is how quantum mechanical aspects of a physical system leave their mark on game-theoretic solutions. The possibility of classical construction of a quantum game does not make the question disappear.

\section{Concluding remarks}

The results in this paper can be summarized as follows. To establish a better comparison with EPR-type experiments, a hypothetical physical implementation of a bi-matrix game is developed that uses repeated tosses with four coins. This opens the way to introduce directly the peculiar probabilities involved in the EPR-type experiments, designed to test the Bell-CHSH inequality, into the proposed procedure to play a bi-matrix game.

The argument rests on the result that when perfect correlations exist between two particles that are forwarded to two players, the violation of Bell-CHSH inequality by the predictions of a class of local hidden variable models forces certain probability measures to take negative values. We investigate how this aspect affects a game and its solutions when it is physically implemented using EPR-type set-up. In such a set-up two choices are made available to each player that are taken as their strategies. Players' payoffs depend on the outcomes of repeated measurements and the constants that define the game.

We find that a consistent set of probabilities can be obtained, given in terms of the statistics involved in the four-coin tossing experiment, such that the game between the players is interpretable as a classical bi-matrix game allowing mixed strategies. The proposal is designed in a way that allows, in the next step, to introduce directly the peculiar probabilities emerging in the EPR-type experiments.

We find that when the game is played with perfectly correlated pairs of particles, the players' payoffs are observed to split into two parts; which correspond to the two situations arising when the predictions, of the class of local hidden variable models, do and do not violate the Bell-CHSH inequality, respectively.

Apart from the splitting of the payoffs, we showed that the implementation using perfectly correlated particles distinguishes between two representations of the game that are completely equivalent in the classical context. We observed that the effects on a game-theoretic solution concept, of whether the predictions of the local hidden variable model do or do not violate the Bell-CHSH inequality, is sensitive to the particular representation used for the game.

Acknowledgment: Author is thankful to anonymous referees for giving helpful comments.

\section{References}

[1] L. Vaidman, Found. Phys. 29, 615 (1999).

[2] David A. Meyer, Phys. Rev. Lett. 82 (1999) 1052.

[3] J. Eisert, M. Wilkens, M. Lewenstein, Phys. Rev. Lett. 83 (1999) 3077.

[4] J. Eisert and M. Wilkens, J. Mod. Opt. 47, 2543 (2000).

[5] Simon C. Benjamin, Patrick M. Hayden, Phys. Rev. A 64 (3): 030301, 2001.

[6] Simon C. Benjamin, Patrick M. Hayden, Phys. Rev. Lett. 87 (6): 069801, 2001.

[7] Luca Marinatto, Tullio Weber, Physics Letters A 272, 291 (2000).

[8] S. J. van Enk, R. Pike, Phys Rev A 66, 024306 (2002). 
[9] E. W. Piotrowski, J. Sladkowski, Int. J. Theor. Phys. 42 (2003) 1089-1099.

[10] E. W. Piotrowski, J. Sladkowski, The Next Stage: Quantum Game Theory, in "Mathematical Physics Research at the Cutting Edge", Ed. C. V. Benton, Nova Science Publishers, 2004, 251-272.

[11] Jiangfeng Du, Hui Li, Xiaodong Xu, Mingjun Shi, Jihui Wu, Xianyi Zhou, Rongdian Han, Physical Review Letter 88, 137902 (2002).

[12] Jiangfeng Du, Xiaodong Xu, Hui Li, Xianyi Zhou, Rongdian Han, Fluctuation and Noise Letters, Vol. 2, No. 4 (2002) R189.

[13] Jiangfeng Du, Guanglei Cheng, Hui Li, Int. J. Mod. Phys. B, Vol. 18, Nos. 17-19 (2004) $2552-2558$.

[14] Edward W. Piotrowski, J. Sladkowski, quant-ph/0406129 v1.

[15] Adrian P. Flitney, Derek Abbott, Proc. R. Soc. (London) A 459 (2003) 2463-74.

[16] Adrian P. Flitney, Derek Abbott, Fluct. Noise Lett. 2 (2002) R175-87.

[17] Taksu Cheon, Izumi Tsutsui, quant-ph/0503233 v2.

[18] J. Shimamura, S. K. Ozdemir, F. Morikoshi and N. Imoto, Int. J. Quant. Inf. 2/1, 79 (2004).

[19] Chiu Fan Lee, Neil Johnson, Phys. Rev. A 67, 022311 (2003).

[20] Adrian Flitney, PhD thesis, Adelaide University, 2005, URL: http://thesis.library.adelaide.edu.au/public/adt-SUA20050429.143949/.

[21] Azhar Iqbal, PhD thesis, Quaid-i-Azam University, 2004, quant-ph/0503176 v3.

[22] J. Orlin Grabbe, quant-ph/0506219 v1.

[23] Diederik Aerts, Haroun Amira, Bart D'Hooghe, Andrzej Posiewnik, Jaroslaw Pykacz, quant-ph/0505120 v1.

[24] A.A. Grib, G.N. Parfionov, quant-ph/0502038 v1.

[25] Azhar Iqbal, J. Phys. A 37, 5873 (2004).

[26] See for example, Kai Lai Chung, Farid AitSahlia, Elementary Probability Theory, Fourth Edition, Springer-Verlag, New York, 2003.

[27] A. Einstein, B. Podolsky and N. Rosen, Phys. Rev. 47, 777 (1935).

[28] J. F. Clauser, M. A. Horne, A. Shimony and R. A. Holt, Phys. Rev. Lett. 23, 880 (1969).

[29] J. F. Clauser and A. Shimony, Rep. Prog. Phys. 41, 1881 (1978).

[30] J. S. Bell, Speakable and Unspeakable in Quantum Mechanics (Cambridge University Press, Cambridge, 1987).

[31] A. Peres, Quantum Theory: Concepts and Methods. Kluwer Academic, Dordrecht, 1993.

[32] W. Mückenheim, Lett. Nuovo Cim. 35, 300 (1982).

[33] Yeong Deok Han, Won Young Hwang, In Gyu Koh, Physics Letters, A 221, 283 (1996).

[34] Jose L. Cereceda, quant-ph/0010091 v4.

[35] T. Rothman and E. C. G. Sudarshan, Int. J. Theor. Phys. 40, Number 8, pp. 1525-1543 (2001). 
[36] W. Mückenheim, Phys. Rep. 133, 337 (1986).

[37] R. P. Feynman, Rev. Mod. Phys. 20, 367 (1948).

[38] R. P. Feynman, Int. J. Theor. Phys. 21, 467 (1982).

[39] W. Mückenheim, in Quantum mechanics versus local realism, edited by F. Selleri (Plenum, New York, 1988), p. 345.

[40] Arthur Fine, Phys. Rev. Lett. 48, 291 (1982).

[41] Daniel I. Fivel, Phys. Rev. Lett. 67, 285 (1991).

[42] K. Gustafson, Quantum Computers and Computing, V. 4, No. 2, 2003, Pages 35-55. URL: http://ics.org.ru/pubsfiles/e/420pdf.pdf

[43] W. De. Baere, A. Mann, and M. Revzen, Found. Phys. 29, 67 (1999).

[44] J. D. Malley, Phys. Rev. A 69, 022118 (2004).

[45] James D. Malley, Arthur Fine, quant-ph/0505016 v1. 